\title{
A Prospective Study of Post Traumatic Stress Disorder among COVID 19 survivors at a tertiary care hospital
}

\author{
Kajalpreet Kaur ${ }^{1}$, Disha Vasavada ${ }^{2}$, Parveen Kumar $^{3}$, Vishal Patel ${ }^{4}$, Deepak Tiwari ${ }^{5}$ \\ ${ }^{1}$ Resident doctor, Department of psychiatry, M.P. Shah Medical College Jamnagar \\ ${ }^{2}$ Resident doctor, Department of psychiatry, M.P. Shah Medical College Jamnagar \\ ${ }^{3}$ Resident doctor, Department of psychiatry, M.P. Shah Medical College Jamnagar \\ ${ }^{4}$ Associate Professor, Dr. M.K. Shah Medical College \& Research Center, Ahmadabad \\ ${ }^{5}$ Professor and Head Of Department, Department Of Psychiatry, M.P. Shah Medical College Jamnagar \\ Corresponding author: Parveen Kumar \\ Email-pjakhad@gmail.com
}

\begin{abstract}
Background: The Novel Corona Virus Disease (COVID-19) outbreak is unprecedented and traumatic event for the many people of the world. Post Traumatic Stress Disorder (PTSD) is the commonly encountered mental health problem after experiencing or witnessing a traumatic event. It is predicted that this outbreak have a more profound and widespread psychological impact. The current study is aimed at estimation of the prevalence of PTSD in patients with COVID-19 at two different points of time.

Methodology: A prospective study was carried out from may 2020 to October 2020 at a COVID notified tertiary care centre. Participants were approached using Semi-structured proforma and 'PTSD Symptom Scale Interview (PSS-I)' at the time of discharge and 1 month post-discharge. Descriptive statistics and Chisquare test were used for the analysis.

Results: A total of 440 participants took part in the study. $20.45 \%$ participants at the time of discharge from the hospital, and $18.33 \%$ at one month follow-up met the criteria for PTSD. Male participants and those who stayed in hospital for more than 14 days reported higher prevalence of PTSD.

Conclusion: High prevalence of PTSD was observed in patients with COVID-19 at the time of discharge from the hospital. It is more among those who stayed in Intensive care unit and high dependency unit, and stayed for longer duration in hospital.
\end{abstract}

Keywords: COVID-19, Post traumatic stress disorder, Survivors

(Paper received $-2^{\text {nd }}$ January 2021, Peer review completed $-5^{\text {th }}$ March 2021, Accepted $-8^{\text {th }}$ April 2021)

\section{INTRODUCTION}

Insufficient knowledge about the transmission of SARS-CoV-2 and protective measures such as wearing of face masks in public is associated with anxiety. Different infectious disease outbreaks such as bubonic plague, Asiatic flu and cholera, Middle East respiratory syndrome, and Ebola have been associated with polarization, racism, blame and resultant psychological distress [1]. PTSD is defined as a stress-related disorder that may develop in individuals who have experienced or witnessed a traumatic event or injury [2]. Previous study on severe acute respiratory syndrome (SARS) outbreak survivors estimated $25.6 \%$ rate of PTSD at 30 months' follow-up [3]. High rates of PTSD were also reported during the outbreak of Middle East respiratory syndrome corona virus (MERS-CoV) among both healthcare workers and patients under quarantine [4].

As the outbreak linger on, physical and mental wellbeing of people exposed to it is significantly affected [5]. Rather COVID-19 outbreak would have a more profound and widespread psychological impact as 
compared to earlier outbreaks [6]. This could be due to the direct result of psychopathological sequel of viral infection of the central nervous system (CNS) or through an immune response [7].

Certain vulnerable groups such as frontline healthcare workers were found to have significantly elevated stress levels and a greater association with fatigue, poor sleep, worries about health and fear of social contact. A year later, the perceived stress levels in the same group were associated with higher depression, anxiety and post-traumatic stress scores. Previous study also reported that COVID 19 patients might experience depression, anxiety, insomnia and delirium [8]. Children, adolescents and elderly have also been identified as being more vulnerable to trauma with more than one mechanism of operation and risk factors [9-10].

Studies report the prevalence of COVID-19-related PTSD symptoms from 7\% [11] to 96.2\% [12] among patients before discharge from quarantine. A recent meta-analysis among patients with severe acute respiratory syndrome (SARS) infections reported prevalence of PTSD around 32.2\% [8].

The degree to which mental health problems emerge after trauma may vary depending on the different variables. Therefore, the current study was aimed at the estimation of the prevalence of PTSD at two time points in individuals who had been hospitalized for the treatment of COVID 19.

\section{METHODOLOGY}

A prospective study was carried out from May 2020 to October 2020 at a COVID 19 notified tertiary care canter among patients discharged after recovery from COVID 19. All the Participants were approached, first at the time of discharge and then at one month post-discharge from the hospital. A clinical interview was carried out using a semi-structured proforma containing demographic details and 'PTSD Symptom Scale Interview (PSS-I)' at the time of discharge. One month later, participants approached again during followup; patients who were not able to reach the hospital were contacted via mobile phone. Patients with preexisting psychiatric illness and uncontrolled medical illnesses at the time of admission and discharge were excluded from the study. Written informed consent was taken from all the participants. Ethical approval for the study was taken from the Institutional Ethics Committee.

\section{Demographic details}

Demographic details such as name, age, gender, income, occupation, past history of psychiatric illness, family type, and history of substance use and chronic medical illness were included.

PTSD Symptom Scale Interview (PSS-I): This scale was designed to assess the symptoms of PTSD (symptoms in the past two weeks or since trauma if less than two weeks.). It consists of 17 items, each item corresponding to the 17 symptoms of PTSD. PSSI assesses re-experiencing, avoidance and arousal subscores as well as total PTSD severity score. Participants' answers were recorded on a four point linear scale ranging from 0 (Not at all) to 3 (5 or more times per week/Very much). Symptoms measured were considered as present if they are rated as 1 (Once per week or less/A little) or greater. Total severity scores on the PSS-I are based on sum of the raw items, which ranges from 0 to 51 . This scale shows good concurrent reliability and validity, with test-retest reliabilities ranging from 0.66 to 0.77 over a 1 -month period. A score of 13 or higher indicates likelihood of PTSD [13].

\section{STATISTICAL ANALYSIS}

Data entry and analysis was done using Microsoft excel and Epi Info software. The socio-demographic profile, prevalence of PTSD and different symptoms of it (re-experiencing, avoidance and arousal) have been expressed in terms of frequency and percentage. Chi square test was applied for categorical data such as gender, days of hospital stay, residing with family or alone, different age groups and history of co-morbid medical illness, to find out relation with post-traumatic stress disorder. 


\section{RESULTS AND DISCUSSION}

A total of 440 participants participated in the study. There were $59.10 \%$ males and $40.90 \%$ females. The age of participants ranges from 21- 64 years with a mean age of $44.23 \pm 12.56$ years. Majority of the participants belonged to Hindu religion $96.36 \%$ followed by Muslim $3.18 \%$ and $0.46 \%$ others.

At the time of discharge from the hospital, $20.45 \%$ of the participants met the criteria for PTSD, with $35 \%$ experiencing re-experience symptoms, $29.10 \%$ experiencing avoidance and $47.27 \%$ experiencing arousal symptoms.

Table 1: Distribution of PTSD with different variables at the time of discharge

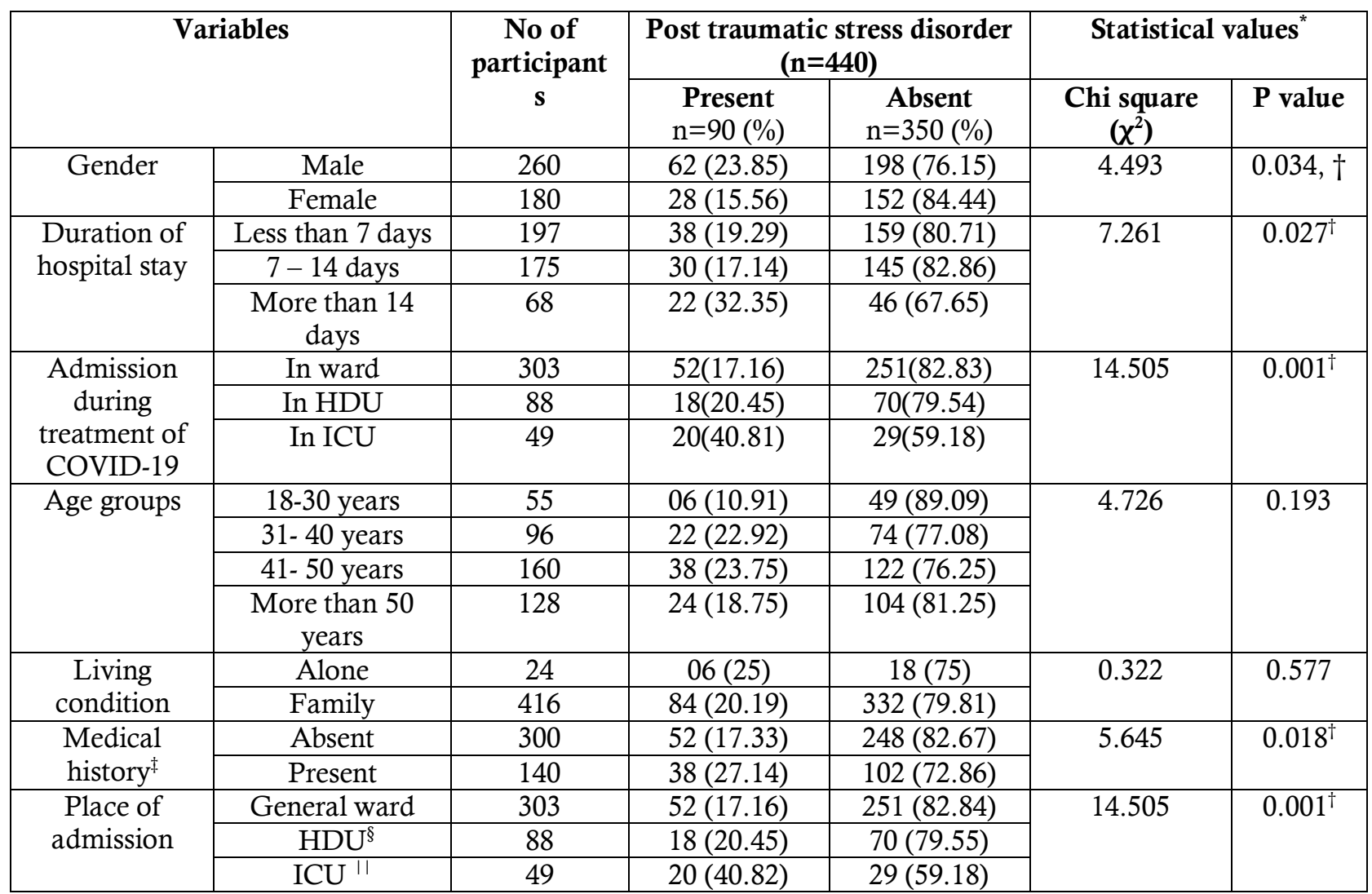

${ }^{*}$ Chi square test, $\uparrow$ Statistical significant, $\ddagger$ Stable on treatment, $\S$ High dependency unit. I Intensive care unit

Table 1 shows that male participants have statistically significant higher prevalence of PTSD than female participants as denoted by chi square test $(\chi 2=4.493, \mathrm{p}=0.034)$. Participants staying in the hospital for more than 14 days had a statistically significant higher prevalence of PTSD, which was denoted by chi square test $(x 2=7.261, p=0.027)$. Participants having any co-morbid medical condition had a statistically higher prevalence of PTSD as shown by chi square test $(\chi 2=5.645, \mathrm{p}=0.018)$. Participants staying in ICU unit of the hospital had a statistically significant higher prevalence of PTSD, which was denoted by chi square test $(\chi 2=14.505, p=0.001)$. No statistically significant difference was observed in the prevalence of PTSD among different age groups and living condition of participants, as shown in table 1.

One month later, 435 participants participated in further study; five participants didn't come for follow-up due to their death. The prevalence of PTSD at one month follow-up was $18.33 \%$. Re-experience symptoms were present in $29 \%$, avoidance symptoms in $19.72 \%$ and arousal symptoms in $39.21 \%$ of the participants.

\section{DISCUSSION}

The current study observed that around 20\% participants had PTSD symptoms at the time of discharge from the hospital. In accordance with these findings, Mazza and others reported 28\% prevalence of PTSD among 
COVID 19 patients by using self-report questionnaires [14]. Mak and others observed $25.6 \%$ prevalence of PTSD among severe acute respiratory syndrome survivors [3]. Roger and others observed $32 \%$ prevalence of PTSD during severe acute respiratory syndrome (SARS) infections [8]. Higher prevalence of PTSD symptoms during COVID 19 pandemic occurs as a result of more stress. The significant psychological stressors contributing to this might be; non-clear evidence about how many cycles of COVID-19 a country may face stigma [15], traumatic memories of severe illness, uncertainty of the future and social isolation [16]. Media also laid great emphasis on the COVID 19 mortality and added fear of death. All these factors could also be possible reasons for higher prevalence of PTSD symptoms in participants with co-morbid medical illness as compared to those without other illnesses in the current study.

The present study observed high prevalence of PTSD among participants with longer duration of hospital stay and participants admitted in ICU unit. Kong and others [17] and Shoar and others [18] observed higher prevalence of psychiatric co-morbidities in patients with longer duration of hospital stay. Tedstone and others [19] and Kangas and his group [20] also observed higher prevalence of PTSD among participants who had experienced life-threatening situations such as admission in intensive care unit (ICU).

These findings are consistent with the view that higher exposure to traumatic events may result in higher rates of morbidity, particularly post-traumatic stress syndromes. Cao and others [21] observed $23.4 \%$ prevalence of PTSD among residents of villages where most houses were destroyed during the Yun Nan (China) earthquake compared to $16.2 \%$ among residents where only minor damage occurred. This demonstrated that prevalence of PTSD was related to the level of exposure to the epicentre of the earthquake [22]. As people were more likely to remember unpleasant events after the disaster, longer duration of hospital stay was associated with more deleterious effect.

The current study observed that male participants had higher level of post-traumatic stress. Chong and others in a study among healthcare workers using impact of event scale at the time of severe acute respiratory syndrome attack observed an increased risk of PTSS among male participants [23] while Rajkumar and others in his study found higher prevalence of post-traumatic stress disorder among female participants during the 2004 Asian tsunami [24]. However, risk factors other than gender may also be operative in a given individual.

No difference in post-traumatic stress among different age groups was observed in the current study. Whereas a study by Wu and others [25] in China and Su and others [26] in Taiwan during SARS outbreak among healthcare workers reported that younger participants had a greater risk of developing PTSS. The results of the current study may differ due to a different study sample or the presence of various comorbidities among different age groups.

The current study observed $18.33 \%$ prevalence of PTSD at one month follow-up. A study by Kawakami and others reported that the mood/anxiety disorders have a greater incidence in the first year post-disaster among survivors and stay for long time [27]. Mak and his group in his study among SARS survivors reported that PTSD was the most prevalent long-term psychiatric condition, even 30 months post-SARS.[3] A Study reported that coping mechanisms used by people may vary and use of different coping mechanisms plays a significant role in the development or maintenance of PTSD [28]. Positive coping attitudes and strong social and family support may have a protective role against acute stress.

The current study was conducted using clinical interview which helps in confirming the diagnosis of PTSD and adds value to the study. However a limitation of this study is that there is no comparison group. Since COVID 19 is a new disease, the psychological effect of some possible long-term physical outcomes related to the disease and treatment regimen has not been discovered until recently. There is a need for further studies to find the true prevalence rate of natural disaster-related PTSD which can then be related to other natural disasters through the use of a similar methodology. Protective factors including resilience should also be explored, which will give us more insight into understanding the coping strategies of those survivors who have not been affected by any kind of mental disorder. Further long term follow up studies are needed to explore the lasting impact as it has been observed that post traumatic symptoms persisted for years after the disaster had ended. Finally the study was carried out at single centre and multicentre studies are needed for better understanding of the phenomenology. 


\section{CONCLUSION}

High prevalence of PTSD was observed at the time of discharge among hospitalized participants. Longer duration of hospital stay is associated with more unpleasant events, subsequently resulting in higher rates of morbidity, particularly post-traumatic stress syndromes. Pre-existing co-morbid conditions further exacerbate the disease outcome. For survivors, a decrease in symptom severity from discharge to one month post-discharge was identified. Exploration of protective factors would give more insight into the understanding of any kind of mental disorder.

\section{REFERENCES}

1. Davtyan, M, Brown, B, Folayan, MO. Addressing Ebola-related stigma: Lessons learned from HIV/AIDS. Global Health Action 2014;7(1):26058.

2. Sareen J. Posttraumatic stress disorder in adults: impact, comorbidity, risk factors, and treatment. Can J Psychiatry 2014;59(9):460-7.

3. Mak IWC, Chu CM, Pan PC, et al.: Long-term psychiatric morbidities among SARS survivors. Gen Hosp Psychiatry 2009;31(4):318-26.

4. Lee SM, Kang WS, Cho A-R, Kim T, Park JK. Psychological impact of the 2015 MERS outbreak on hospital workers and quarantined hemodialysis patients. Compr Psychiatry 2018;87(11):123-7.

5. Norris FH, Friedman MJ, Watson PJ, Byrne CM, Diaz E, Kaniasty K. 60,000 disaster victims speak: Part I. An empirical review of the empirical literature, 1981-2001. Psychiatry 2002;65(3):207-39.

6. Jakovljevic M, Bjedov S, Jaksic N, Jakovljevic I. COVID-19 Pandemia and Public and Global Mental Health from the Perspective of Global Health Securit. Psychiatr Danub 2020;32(1):6-14.

7. Wu Y, Xu X, Chen Z, Duan J, Hashimoto K, Yang L, et al. Nervous system involvement after infection with COVID-19 and other coronaviruses. Brain Behav Immun 2020;87(1):18-22.

8. Rogers JP, Chesney E, Oliver D, Pollak TA, McGuire P, Fusar-Poli P, et al. Psychiatric and neuropsychiatric presentations associated with severe coronavirus infections: a systematic review and meta-analysis with comparison to the COVID-19 pandemic. The Lancet Psychiatry 2020;7(7):611-27.

9. Bhopal SS, Bagaria J, Bhopal R. Risks to children during the covid-19 pandemic: some essential epidemiology. BMJ 2020;369:m2290.

10. Yang Y, Li W, Zhang Q, Zhang L, Cheung T, Xiang Y-T. Mental health services for older adults in China during the COVID-19 outbreak. Lancet Psychiatry 2020;7(4):e19.

11. Liu N, Zhang F, Wei C, Jia Y, Shang Z, Sun L, et al. Prevalence and predictors of PTSS during COVID-19 outbreak in China hardest-hit areas: Gender differences matter. Psychiatry Res 2020;287:112921.

12. Bo H-X, Li W, Yang Y, Wang Y, Zhang Q, Cheung T, et al. Posttraumatic stress symptoms and attitude toward crisis mental health services among clinically stable patients with COVID-19 in China. Psychol Med 2020;27:1-2.

13. Foa EB, Riggs DS, Dancu CV, Rothbaum BO. Reliability and validity of a brief instrument for assessing posttraumatic stress disorder. J Traum Stress 1993;6(4):459-73.

14. Mazza MG, De Lorenzo R, Conte C, Poletti S, Vai B, Bollettini I, et al. Anxiety and depression in COVID19 survivors: Role of inflammatory and clinical predictors. Brain Behav Immun 2020;89:594-600.

15. Tandon R. The COVID-19 pandemic, personal reflections on editorial responsibility. Asian J Psychiatry 2020;50:102100.

16. Brooks SK, Webster RK, Smith LE, Woodland L, Wessely S, Greenberg N, et al. The psychological impact of quarantine and how to reduce it: rapid review of the evidence. The Lancet 2020;395(10227):912-20.

17. Kong X, Zheng K, Tang M, Kong F, Zhou J, Diao L, et al. Prevalence and Factors Associated with Depression and Anxiety of Hospitalized Patients with COVID-19. medRxiv 2020;2020.03.24.20043075.

18. Shoar S, Naderan M, Aghajani M, Sahimi-Izadian E, Hosseini-Araghi N, Khorgami Z. Prevalence and Determinants of Depression and Anxiety Symptoms in Surgical Patients. Oman Med J 2016;31(3):176-81.

19. Tedstone JE, Tarrier N. Posttraumatic stress disorder following medical illness and treatment. Clin Psychol Rev 2003;23(3):409-48.

20. Kangas M, Henry JL, Bryant RA. Posttraumatic stress disorder following cancer. A conceptual and empirical review. Clin Psychol Rev 2002;22(4):499-524.

21. Cao H, McFarlane AC, Klimidis S. Prevalence of psychiatric disorder following the 1988 Yun Nan (China) earthquake--the first 5-month period. Soc Psychiatry Psychiatr Epidemiol 2003;38(4):204-12.

22. Chong M-Y, Wang W-C, Hsieh W-C, Lee C-Y, Chiu N-M, Yeh W-C, et al. Psychological impact of severe acute respiratory syndrome on health workers in a tertiary hospital. Br J Psychiatry 2004;185:127-33.

23. Rajkumar AP, Mohan TSP, Tharyan P. Lessons from the 2004 Asian tsunami: epidemiological and nosological debates in the diagnosis of post-traumatic stress disorder in non-Western post-disaster communities. Int J Soc Psychiatry 2013;59(2):123-9.

24. Wu P, Fang Y, Guan Z, Fan B, Kong J, Yao Z, et al. The psychological impact of the SARS epidemic on hospital employees in China: exposure, risk perception, and altruistic acceptance of risk. Can J Psychiatry 2009;54(5):302-11. 
25. Su T-P, Lien T-C, Yang C-Y, Su YL, Wang J-H, Tsai S-L, et al. Prevalence of psychiatric morbidity and psychological adaptation of the nurses in a structured SARS caring unit during outbreak: a prospective and periodic assessment study in Taiwan. J Psychiatr Res 2007;41(1-2):119-30.

26. Kawakami N, Fukasawa M, Sakata K, Suzuki R, Tomita H, Nemoto H, et al. Onset and remission of common mental disorders among adults living in temporary housing for three years after the triple disaster in Northeast Japan: comparisons with the general population. BMC Pub Health 2020;1271.

27. Voges MA, Romney DM. Risk and resiliency factors in posttraumatic stress disorder. Ann Gen Hosp Psychiatry 2003;2(1):4.

$* * * * * * * * * * * * * * * * * * * * * * * * * * * * * * * * * * * *$

Acknowledgements - Nil

Conflict of Interest - Nil

Funding - Nil 